\title{
Synthesis and Crystallography of 1-acetoxy-3-ethoxy carbonyl-4-(4-methoxy)phenyl-7-methoxynaphthalene
}

\author{
Ashok Kumar Singh* \\ Department of Chemistry, Tri-Chandra Multiple Campus, Tribhuvan University, Nepal \\ Email: asokksingh@yahoo.co.in
}

\begin{abstract}
1-Acetoxy-3-ethoxy carbonyl-4-(4-methoxy)phenyl-7-methoxynaphthalene (3) has been synthesized and characterized by $X$-ray diffraction method. The compound crystallizes in triclinic space group $P-1$ with cell parameter $a=7.793$ (6) $\dot{A}, b=10.7428(16) \dot{A}, c=13.306$ (4) $\dot{A}$ and $Z=2$.
\end{abstract}

Keywords: Phenyl naphthalene, aryl naphthalene, ligran, Benzophenone, diethylsuccinate

\section{Introduction}

Several aryl-1,2-dihydronaphthalene ${ }^{1}$ and aryl naphthalenes which are derivatives of lignans exhibit inhibitory activity against viral reverse transcriptase, such as retrojusticidin and phyllamyricin ${ }^{2}$ . Also they exhibit hypolipidemic activity ${ }^{3}$. Kamal et al. ${ }^{4}$ have carried out the preparation of dehydropodophyllotox in an important representative of arylnaphthalene lignans, in the presence of yeast. Owing to their interest as antineoplastic agents ${ }^{5}$ and other pharmacological activities, ${ }^{6}$ considerable work has been completed on lignans and other derivatives. In view of these significances in this article we report the synthesis of some naphthalene analogues. In retrospect of the above observations, the presentation is directed towards facile synthesis and $\mathrm{x}$-ray crystallographic studies of [1-acetoxy-3-ethoxy carbonyl-4-(4-methoxy)phenyl-7-methoxynaphthalene.

\section{Experimental}

In a typical procedure, to freshly prepared potassium tertiary butoxide $(1.56 \mathrm{~g}$ of potassium in $60 \mathrm{~mL}$ of t-butanol) 4,4'-dimethoxybenzophenone 1 was added quickly under nitrogen and refluxed for $1 \mathrm{~h}$. To this mixture freshly distilled diethylsuccinate $(6.6 \mathrm{~mL}, 0.04 \mathrm{~mol})$ was added at once and refluxed for $30 \mathrm{~h}$. The excess of t-butanol was removed by distillation under reduced pressure and the residue was acidified with $5 \mathrm{~N} \mathrm{HCl}$. The product was extracted into $10 \% \mathrm{NaHCO}_{3}$ solution and washed with diethyl ether $(3 \times 20$ $\mathrm{mL}$ ). Finally on acidification with $10 \% \mathrm{HCl}$, it gave isomers of $\mathbf{2}$ as yellow pasty mass. 2.

In a typical procedure, a mixture of $2(5 \mathrm{~g}, 0.01 \mathrm{~mol})$, fused sodium acetate $(1.38 \mathrm{~g}, 0.016 \mathrm{~mol})$ and acetic anhydride $(41.4 \mathrm{~mL})$ was stirred over night at room temperature. It was refluxed at $80{ }^{\circ} \mathrm{C}$ for $5 \mathrm{~h}$. The crude product was extracted in ether $(3 \times 20 \mathrm{~mL})$ and washed with distilled water $(3 \times 30 \mathrm{~mL})$ to remove excess of sodium acetate and acetic anhydride. Finally it was washed with $10 \% \mathrm{NaHCO}_{3} 10 \%$ $\mathrm{NaOH}$ and distilled water and dried over sodium sulphate. After evaporation the product was recrystallized with ethanol. gave 1-acetoxy-3-ethoxy carbonyl-4-(4-methoxy)phenyl-7methoxynaphthalene (3) in \% ( g) yield. M.P $95-96{ }^{\circ} \mathrm{C}$.

\section{* Corresponding author}




\section{Results and Discussion}

IR (Neat) of yellow pasty mass. 2 reveals the following information: $1660(\mathrm{C}=\mathrm{O}), 1712(\mathrm{C}=\mathrm{O}$ of ester), 1600 (C=O of acid), 3100-3200 $\mathrm{cm}^{-1}$ (OH of carboxylic acid); ${ }^{1} \mathrm{H}$ NMR $\left(\mathrm{CDCl}_{3}\right): \delta 1.02(\mathrm{t}, 3 \mathrm{H}$, $\left.\mathrm{CH}_{3}\right), 1.9-2.3\left(\mathrm{~m}, 2 \mathrm{H}, \mathrm{CH}_{2}\right) 0.9\left(\mathrm{t}, \mathrm{J}=6 \mathrm{~Hz}, 3 \mathrm{H}, \mathrm{CH}_{3}\right), 3.3$ (bs, $\left.2 \mathrm{H}, \mathrm{CH}_{2}-\mathrm{C}=\mathrm{O}\right), 3.75\left(\mathrm{~s}, 6 \mathrm{H}, 2 \mathrm{OCH}_{3}\right) 3.9$ (q, $\left.2 \mathrm{H}, \mathrm{OCH}_{2}\right), 6.8-7.5(\mathrm{~m}, 9 \mathrm{H}, \mathrm{Ar}-\mathrm{H}), 10.5$ (bs, $\left.1 \mathrm{H}, \mathrm{COOH}\right)$.

IR(Nujol) 1-acetoxy-3-ethoxy carbonyl-4-(4-methoxy)phenyl-7-methoxynaphthalene (3) reveals the following information: $1660(\mathrm{C}=\mathrm{O}), 1765 \mathrm{~cm}^{-1}(\mathrm{C}=\mathrm{O}$ of ester $) ;{ }^{1} \mathrm{H} \mathrm{NMR}\left(\mathrm{CDCl}_{3}\right): \delta$ 0.9-1.1 (t, $3 \mathrm{H}, \mathrm{CH}_{3}$ of ethyl ), 2.5 (s, $3 \mathrm{H}, \mathrm{CH}_{3}$ ), 3.8 (s, $6 \mathrm{H}, 2 \mathrm{OCH}_{3}$ ), 3.9-4.15(q, $\left.2 \mathrm{H}, \mathrm{CH}_{2}\right), 6.8-7.7$ (m ,8H, Ar-H).

A view of (3), showing the atom-numbering scheme, is given in Fig. 1 and selected geometric parameters are given in Table 1.

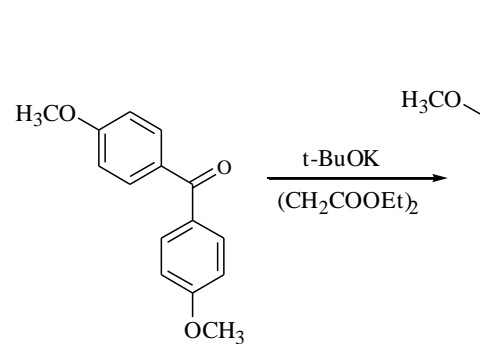

1

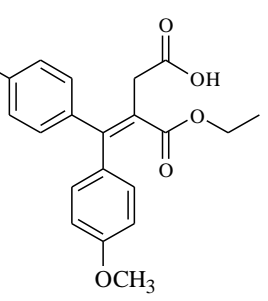

2

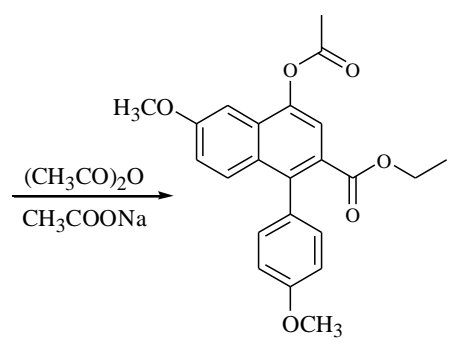

3

Scheme 1

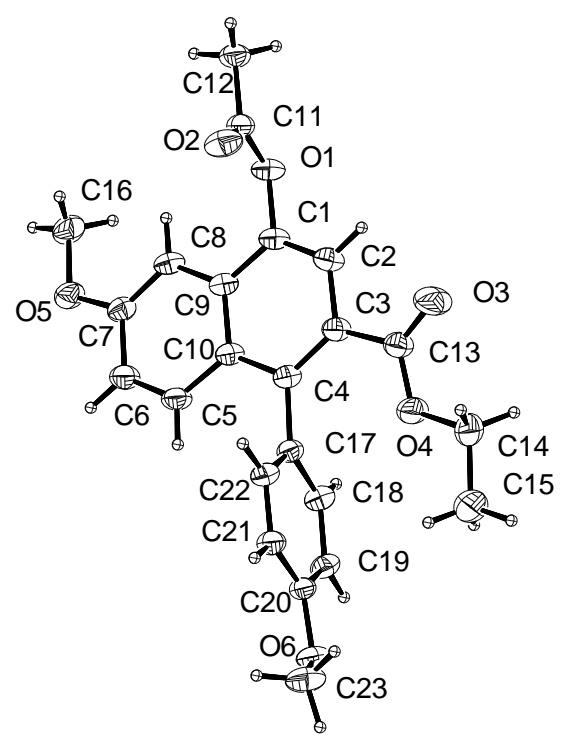

Figure 1: Triclinic Crystal of compound 3 
Table 1: Geometric parameters

\begin{tabular}{ll}
\hline Formula & $\mathrm{C}_{23} \mathrm{H}_{22} \mathrm{O}_{6}$ \\
Formula weight & 394.41 \\
Crystal system & triclinic \\
Space group & $\mathrm{P}_{-}-1, \mathrm{Z}=2$ \\
$\mathrm{a}$ & $7.793(6) \AA$ \\
$\mathrm{b}$ & $10.7428(16) \AA$ \\
$\mathrm{c}$ & $13.306(4) \AA$ \\
$\alpha$ & $71.097(18)$ \\
$\beta$ & $74.94(3)^{0}$ \\
$\nu$ & $88.43(3) \AA^{3}$ \\
$\mathrm{D}_{\mathrm{x}}$ & $1.290 \mathrm{Mg} / \mathrm{m}^{3}$ \\
$2 \theta_{\max }$ & $49.44^{0} \mathrm{with} \mathrm{MoKa}$ \\
No. of reflections used $(\mathrm{I}>2 \sigma(\mathrm{I})$ & 2422 \\
$\mathrm{R}$ & 0.0447 \\
$(\Delta / \sigma)_{\max }$ & 0.000 \\
$(\Delta \rho)_{\max }$ & $0.996 \mathrm{e} \AA$ \\
$(\Delta \rho)_{\min }$ & $-0.189 \mathrm{e} \AA$ \\
Measurement & Enraf-Nonius MACH3 diffractometer \\
Program system & SHELXS-97 \\
Refinement fullmatrix: & SHELXS-97 \\
\hline
\end{tabular}

Table 2: Atomic coordinates and equivalent temperature factors $\left(\hat{A}^{2}\right)$

\begin{tabular}{|c|c|c|c|c|}
\hline Atom & $\mathrm{x}$ & $\mathrm{y}$ & $\mathrm{Z}$ & $\mathrm{U}_{\mathrm{eq}}$ \\
\hline $\mathrm{O} 1$ & $0.41070(19)$ & $0.88485(12)$ & $0.22273(13)$ & $0.0633(4)$ \\
\hline $\mathrm{O} 2$ & $0.2812(2)$ & $0.97064(15)$ & $0.08538(14)$ & $0.0792(5)$ \\
\hline O3 & $-0.1818(2)$ & $0.64003(17)$ & $0.39058(19)$ & $0.1019(7)$ \\
\hline O4 & $-0.13711(19)$ & $0.43258(14)$ & $0.40573(14)$ & $0.0746(5)$ \\
\hline O5 & $0.93619(18)$ & $0.65860(14)$ & $0.05142(13)$ & $0.0667(4)$ \\
\hline O6 & $-0.0388(2)$ & $-0.01032(12)$ & $0.33408(13)$ & $0.0688(4)$ \\
\hline $\mathrm{C} 1$ & $0.3457(3)$ & $0.75738(18)$ & $0.23785(18)$ & $0.0559(5)$ \\
\hline $\mathrm{C} 2$ & $0.1755(3)$ & $0.71636(19)$ & $0.29434(19)$ & $0.0607(5)$ \\
\hline $\mathrm{C} 3$ & $0.1089(3)$ & $0.58774(18)$ & $0.31207(17)$ & $0.0556(5)$ \\
\hline $\mathrm{C} 4$ & $0.2189(3)$ & $0.50278(18)$ & $0.27015(16)$ & $0.0520(5)$ \\
\hline $\mathrm{C} 5$ & $0.5197(3)$ & $0.46689(19)$ & $0.16249(18)$ & $0.0603(6)$ \\
\hline C6 & $0.6918(3)$ & $0.5081(2)$ & $0.10970(19)$ & $0.0632(6)$ \\
\hline C7 & $0.7585(3)$ & $0.63211(19)$ & $0.10108(17)$ & $0.0561(5)$ \\
\hline $\mathrm{C} 8$ & $0.6472(3)$ & $0.71547(18)$ & $0.14130(17)$ & $0.0570(5)$ \\
\hline C9 & $0.4668(3)$ & $0.67510(18)$ & $0.19560(17)$ & $0.0527(5)$ \\
\hline $\mathrm{C} 10$ & $0.4001(3)$ & $0.54687(17)$ & $0.21029(17)$ & $0.0526(5)$ \\
\hline $\mathrm{C} 11$ & $0.3708(3)$ & $0.98600(19)$ & $0.14087(18)$ & $0.0559(5)$ \\
\hline $\mathrm{C} 12$ & $0.4545(3)$ & $1.11272(19)$ & $0.1320(2)$ & $0.0662(6)$ \\
\hline $\mathrm{C} 13$ & $-0.0836(3)$ & $0.5572(2)$ & $0.37303(19)$ & $0.0611(5)$ \\
\hline $\mathrm{C} 14$ & $-0.3256(3)$ & $0.3980(2)$ & $0.4579(2)$ & $0.0756(7)$ \\
\hline $\mathrm{C} 15$ & $-0.3574(4)$ & $0.2558(3)$ & $0.4775(3)$ & $0.0986(9)$ \\
\hline C16 & $1.0147(3)$ & $0.7770(2)$ & $0.0510(2)$ & $0.0772(7)$ \\
\hline $\mathrm{C} 17$ & $0.1573(2)$ & $0.36706(17)$ & $0.28193(16)$ & $0.0499(5)$ \\
\hline $\mathrm{C} 18$ & $0.1861(3)$ & $0.25812(19)$ & $0.36551(17)$ & $0.0573(5)$ \\
\hline C19 & $0.1224(3)$ & $0.13414(19)$ & $0.37892(18)$ & $0.0578(5)$ \\
\hline $\mathrm{C} 20$ & $0.0255(3)$ & $0.11647(17)$ & $0.31100(17)$ & $0.0524(5)$ \\
\hline $\mathrm{C} 21$ & $0.0002(3)$ & $0.22260(18)$ & $0.22618(17)$ & $0.0568(5)$ \\
\hline $\mathrm{C} 22$ & $0.0679(3)$ & $0.34659(18)$ & $0.21198(17)$ & $0.0551(5)$. \\
\hline $\mathrm{C} 23$ & $-0.1701(4)$ & $-0.0284(2)$ & $0.2831(2)$ & $0.0863(8)$ \\
\hline
\end{tabular}

$\mathrm{U}_{\mathrm{eq}}=(1 / 3) \Sigma_{\mathrm{i}} \Sigma_{\mathrm{j}} \mathrm{U}_{\mathrm{ij}}\left(\mathrm{a}_{\mathrm{i}}^{*} \mathrm{a}_{\mathrm{j}}^{*}\right)\left(\mathrm{a}_{\mathrm{i}} \mathrm{a}_{\mathrm{j}}\right)$ 
J. Nepal Chem. Soc., vol. 28, 2011

Table 3 Selected bond lengths $(\dot{A})$ and bond angles $\left(^{\circ}\right)$

\begin{tabular}{|c|c|c|}
\hline O1-C11 1.362(3) & C11-O1-C1 & $117.07(16)$ \\
\hline $\mathrm{O} 1-\mathrm{C} 1 \quad 1.407(2)$ & C13-O4-C14 & $117.38(18)$ \\
\hline $\mathrm{O} 2-\mathrm{C} 11 \quad 1.189(3)$ & C7-O5-C16 & $117.15(18)$ \\
\hline O3-C13 1.198(3) & C20-O6-C23 & $117.48(17)$ \\
\hline $\mathrm{O} 4-\mathrm{C} 13 \quad 1.310(2)$ & $\mathrm{C} 2-\mathrm{C} 1-\mathrm{O} 1$ & $120.12(18)$ \\
\hline $\mathrm{O} 4-\mathrm{C} 14 \quad 1.453(3)$ & $\mathrm{C} 2-\mathrm{C} 1-\mathrm{C} 9$ & $122.48(18)$ \\
\hline O5-C7 1.364(3) & $\mathrm{O} 1-\mathrm{C} 1-\mathrm{C} 9$ & $117.36(18)$ \\
\hline O5-C16 1.424(2) & $\mathrm{C} 1-\mathrm{C} 2-\mathrm{C} 3$ & $120.9(2)$ \\
\hline O6-C20 $1.374(2)$ & $\mathrm{C} 4-\mathrm{C} 3-\mathrm{C} 2$ & 119.81(19) \\
\hline O6-C23 1.415(3) & C4-C3-C13 & $125.12(18)$ \\
\hline C1-C9 $1.410(3)$ & C2-C3-C13 & $115.03(18)$ \\
\hline C2-C3 1.414(3) & C3-C4-C10 & 119.07(17) \\
\hline C3-C4 1.385(3) & C3-C4-C17 & $123.16(18)$ \\
\hline C3-C13 1.497(3) & C10-C4-C17 & 117.76(17) \\
\hline C4-C10 $1.435(3)$ & $\mathrm{O} 5-\mathrm{C} 7-\mathrm{C} 8$ & $125.18(18)$ \\
\hline C4-C17 1.494(3) & O5-C7-C6 & $114.86(19)$ \\
\hline C6-C7 1.402(3) & C8-C7-C6 & 119.96(19) \\
\hline C9-C10 1.421(3) & C1-C9-C8 & $122.62(18)$ \\
\hline C11-C12 1.483(3) & $\mathrm{C} 1-\mathrm{C} 9-\mathrm{C} 10$ & 117.11(19) \\
\hline C14-C15 1.480(3) & $\mathrm{C} 5-\mathrm{C} 10-\mathrm{C} 4$ & $122.44(17)$ \\
\hline C17-C22 1.370(3) & C9-C10-C4 & $120.60(18)$ \\
\hline C19-C20 1.377(3) & $\mathrm{O} 2-\mathrm{C} 11-\mathrm{O} 1$ & $122.86(18)$ \\
\hline \multirow[t]{11}{*}{ C20-C21 1.372(3) } & $\mathrm{O} 2-\mathrm{C} 11-\mathrm{C} 12$ & $126.4(2)$ \\
\hline & $\mathrm{O} 1-\mathrm{C} 11-\mathrm{C} 12$ & $110.74(19)$ \\
\hline & O3-C13-O4 & $122.4(2)$ \\
\hline & $\mathrm{O} 3-\mathrm{C} 13-\mathrm{C} 3$ & $122.8(2)$ \\
\hline & $\mathrm{O} 4-\mathrm{C} 13-\mathrm{C} 3$ & $114.76(18)$ \\
\hline & O4-C14-C15 & $107.8(2)$ \\
\hline & C22-C17-C18 & 118.04(17) \\
\hline & C22-C17-C4 & $121.05(17)$ \\
\hline & C21-C20-O6 & $124.45(19)$ \\
\hline & C21-C20-C19 & $119.60(17)$ \\
\hline & O6-C20-C19 & $115.95(17)$ \\
\hline
\end{tabular}

\section{Acknowledgements}

E. Balaram of department of chemistry, university of Hyderabad is acknowledged for helping with XRD.

\section{References}

1. P. K. Datta, C. Yau, T. Hooper, S. Brigitte, L.Y. James. L. Charlton, J Org Chem, , 2001, 66, 8606; S. Brigitte, Probal K. Datta, Trung N. Le, L. James, L. Charlton, Synthesis, 2001, 66,1556.

2. S.R. Ward, Nat. Prod Rep, 1999, 16, 75; Lee C. T. L. \& Lin V. C. K, Bioorg Med Chem Lett, 1997, 7, 2897.

3. T. Iwasaki, K. Kondo, T. Nishitani, T. Kuroda, K. Hirakoso, A. Ohtani, K. Takashima, Chem Pharm Bull, 1995, 43, 1701.

4. A. Kamal, and Y. Damayanthi, Bioorg Med Chem Lett, 1997, 7, 657.

5. I. Jardine, Podophyllotoxins in Anticancer agents Based on Natural Products Models, Academic Press Inc. New York, 1980, p.319.

6. W.D MacRae, and G.H.N. Towers, Phytochemistry, 1984, 23, 1207; W.D. MacRae, J.B Hudson and G.H.N Towers, Planta Me., 1989, 55, 531. 